\title{
Study of the genetic diversity of maize samples with dark colored grains from the gene pool of Azerbaijan
}

\author{
Valiyeva L.S.*, Ragimova G.K., Nabiyeva N.A. \\ Genetic Resources Institute, ANAS, Baku, Azerbaijan \\ *e-mail: l.valiyeva@yandex.ru
}

In the corn grain (Zea mays L.), flavonoid grouping and possessing many health properties (antioxidant, antimicrobial, anti-carcinogenic) anthocyanin pigments can accumulate. Numerous studies have found that regular consumption of food rich in anthocyanins leads to a significant decrease in the level of diabetes, obesity, cardiovascular and oncological diseases. The beneficial effect is manifested depending on the type and amount of anthocyanins in the ration component. The combination of nutritional and therapeutic value of the rich content of useful anthocyanins of corn kernels characterizes it as a functional product. The accumulation of anthocyanins in corn grain is controlled by the coordinated expression of structural and regulatory genes, the combination of different allelic variants of which determines the quantity and quality of the anthocyanins synthesized. Out of more than 400 maize samples from the National Gene Bank of Azerbaijan, forms with dark-colored grains were selected to study and use their genetic potential in breeding. The purpose of this work was to study the genetic diversity of maize samples with dark colored grains adapted to local conditions for further selection on grain quality. The paper presents the results of genotyping 38 maize samples using intermicrosatellite ISSR primers selected from the literature (inter simple sequence repeats), the most widely used and effective classes of DNA markers for genotyping, certification and classification of plant varieties. 6 primers generated polymorphic, well reproducible PCR fragments. For each sample, individual ISSR spectra were obtained, differing in the number of amplicons. A total of 65 fragments were synthesized, of which 63 are polymorphic. Thus, the average level of polymorphism in the 6 primers used was $96.9 \%$, which indicates a wide range of genetic variability in the sample under study, the samples of which can serve as the starting material for the selection of corn to improve the qualitative and quantitative composition of anthocyanins in the grain. 